\title{
REDUCING THE NUMBER OF VARIABLE MOVEMENTS IN EXACT BDD MINIMIZATION
}

\author{
Rüdiger Ebendt \\ Department of Computer Science \\ University of Kaiserslautern \\ 67663 Kaiserslautern, Germany \\ ebendt@informatik.uni-kl.de
}

\begin{abstract}
Ordered Binary Decision Diagrams (BDDs) are frequently used in logic synthesis.

In this paper a new exact BDD minimization algorithm is presented, which is based on state space search. In contrast to all previous approaches, in which variables are moved through the BDD when exploring the state space, the new method makes use of a new technique to expand states to its successor states without expensive variable movements. Experimental results are given to show the efficiency of the approach.
\end{abstract}

\section{INTRODUCTION}

Recently, the interest in logic synthesis based on FPGAs and Pass Transistor Logic (PTL) has been renewed. These techniques and the resulting circuits have several desirable properties, amongst them improved power dissipation, circuit speed and area as well as high design quality by consideration of layout aspects during synthesis $[15,3,7,14,13,5]$. These techniques make use of ordered Binary Decision Diagrams (BDDs) as introduced in [2]. Their size often critically depends on the chosen variable ordering.

In applications like logic synthesis it is most important to determine a good ordering, since a reduction in the number of BDD nodes directly transfers to a smaller chip area.

Heuristical approaches $[9,16]$ cannot guarantee an optimal result and often yield BDDs up to twice the size of the best known solution. Since this is a serious problem, exact algorithms have been proposed $[8,11,12,4,6]$. Due to the NP-completeness of the problem of improving a given variable ordering [1], all suggested approaches so far have longer runtimes compared to heuristics. A main reason for the long runtimes of all known approaches is that they require many time-consuming movements of variables through the BDD. Hence, there is a strong demand for faster solutions.

In this paper a new exact algorithm for the computation of an optimal variable ordering is presented. As all other exact algorithms presented so far, the core technique is based on [8] in combination with branch\&bound. But in contrast to previous approaches, a new state expansion technique without expensive movement of variables through the BDD is used. Experiments show that significant runtime reductions can be observed on benchmark functions, i.e. for the functions considered an improvement of up to $63.9 \%$ has been obtained.

\section{PRELIMINARIES}

Boolean variables (denoted by Latin letters) are bound to values in $\mathbf{B}:=\{0,1\}$. It is well-known that a Boolean function $f: \mathbf{B}^{n} \rightarrow \mathbf{B}$ over the variable set $X_{n}$ can be represented by a $B i$ nary Decision Diagram (BDD) [2], i.e. a directed acyclic graph where a Shannon decomposition

$$
f=x_{i} f_{x_{i}=1}+\bar{x}_{i} f_{x_{i}=0} \quad(1 \leq i \leq n)
$$

into two cofactors in $x_{i}$ is carried out in each node, yielding a "then-successor" via a 1-edge and an "else-successor" via a 0edge (as an example for a BDD see Figure 2, the annotated variable sets for now can be ignored and are explained later in Section 4.1). In the following, only reduced, ordered BDDs are considered and for briefness these graphs are called BDDs. Redundant nodes are assumed to be eliminated and variables are encountered at most once and in the same order (the "variable ordering", e.g. $x_{1}<x_{2}<x_{3}<x_{4}$ in Figure 2) on every path from the root to a terminal node. For more details see [2]. Variable orderings are often denoted by permutations $\pi$. For simplicity, the author uses the notation $x_{i}=\pi(k)$, if variable $x_{i}$ is the $k$-th element of the variable ordering, i.e. $x_{i}$ is in the $k$-th level of the BDD. Permutations $\pi$ are extended straightforwardly to also map subsets of $X_{n}$ to subsets of $X_{n}$. Further, the minimal number of nodes in a BDD labeled by a variable in $I \subseteq X_{n}$ is denoted with min_cost $t_{I}$. BDDs are defined analogously for multi-output functions $f: \mathbf{B}^{n} \rightarrow \mathbf{B}^{m}$, using a graph for each of the $m$ singleoutput functions $\left(f_{i}^{n}\right)_{1<i<m}$ for the shared BDD representation. In the following shared BDDs with Complement Edges (CEs) are assumed without mentioning it further. (Note that all results reported here directly transfer to BDDs without CEs.) For a BDD $F$, let label $\left(F, x_{k}\right)$ denote the number of nodes in $F$ labeled by $x_{k}$.

\section{PREVIOUS WORK}

To keep the paper self-contained, the fastest approaches to exact BDD minimization known so far $[4,6]$, which are based on the framework of [11], are briefly reviewed.

Suppose the BDD for a multi-output function $f: \mathbf{B}^{n} \rightarrow \mathbf{B}^{m}$ is minimized. In brief, the optimal variable ordering is computed iteratively by computing for increasing $k$ 's min_cost $_{I}$ for each $k$ element subset $I$ of $X_{n}$, until $k=n$ : then, the BDD has a variable ordering yielding a BDD size of min_cost $X_{n}$. This is an optimal variable ordering. 
At step $k$ of the algorithm, a state $I$ with $|I|=k-1$ is retrieved from a hash table (which holds all states of the previous step $k-1$ ). The algorithm now builds transitions $I \stackrel{x_{i}}{\longrightarrow} I \cup\left\{x_{i}\right\}=: I^{\prime}$ for $x_{i} \in X_{n} \backslash I$. The subject is to compute min_cost $I_{I^{\prime}}$ for each successor $I^{\prime}$. This is done by a gradual scheme of continuous minimum updates following [8], which computes min_cost $_{I^{\prime}}$ by use of the minimum values for the predecessors of $I^{\prime}$. These values have been computed in the last step $k-1$ and are simply retrieved from a hash table. Therefore, following [4, 6], the only terms needed to compute for a state $I$, which is going to be expanded in step $k$ are label $\left(F_{i}, x_{i}\right)$ for each $x_{i} \in X_{n} \backslash I$, where $F_{i}$ is a BDD representing $f$ with variable ordering $\pi_{i}$ such that $\pi_{i}(I)=I$ and $\pi_{i}(|I|+1)=x_{i}$.

At the end of step $k$, all states for which a lower bound on the BDD size achievable from that state exceeds or equals the current known upper bound on the minimal BDD size, are excluded. In [4] an effective lower bound known from VLSI design was used and in [6], three lower bounds were used in parallel, further enhancing this approach. For more details on exact BDD minimization with branch\&bound see $[11,4,6]$.

\section{ALGORITHM}

In this section the state expansion technique used in the new approach is described. The implementation of the algorithm is based on the implementation of recent approaches $[4,6]$. Hence the new method uses techniques, that have been applied there. Amongst them are the use of symmetries, customized hash tables and the efficient technique for fast BDD reconstruction of [6]. The presence of all these techniques is assumed without further mentioning. A sketch of the new algorithm called NEO is given in Figure 1. With "hash()" the use of the double hash function as suggested in [4] is indicated. It is applied both to variable sets, implemented as bitmasks of $n$ bits which are interpreted as longword size integers and to the addresses of BDD nodes, interpreted as word size integers.

\subsection{Theoretical Background and Idea}

Reconsidering the exact minimization algorithm described in section 3, it can be observed: In step $k$, the algorithm expands all states $I$ with $|I|=k-1$ to all possible successor states $I^{\prime}=$ $I \cup\left\{x_{i}\right\}$ for $x_{i} \in X_{n} \backslash I$. The terms $\operatorname{label}\left(F_{i}, x_{i}\right)$ (where $F_{i}$ is a BDD representing $f$ with variable ordering $\pi_{i}$ such that $\pi_{i}(I)=I$ and $\pi_{i}\left(\left|I^{\prime}\right|\right)=x_{i}$ ) now must be computed. In previous approaches this was done by actually constructing the BDDs $F_{i}$ requiring (at least) the movement of the variables $x_{i}$ through the BDD to the $(|I|+1)$-th level. This is a very time consuming operation with the potential risk of increasing the number of BDD nodes ("BDD explosion"). The new approach applies a different technique for this, using an argument, which follows [8]:

Let $I \subseteq X_{n}$ and $x_{i} \in X_{n} \backslash I$. Let $F$ be a BDD with a variable ordering $\pi$ such that $\pi(I)=I$ and $\pi(|I|+1)=x_{i}$. Assume, $F$ is representing a multi-output function $f: \mathbf{B}^{n} \rightarrow \mathbf{B}^{m}$ which can be written as family $\left(f_{i}{ }^{n}\right)_{1 \leq i \leq m}$. Let $\operatorname{dep}\left(f, I, x_{i}\right)$ denote the set of those cofactors of the functions $\left(f_{i}{ }^{n}\right)_{1 \leq i \leq m}$ in all variables in $I$, which are functions depending essentially on $x_{i}$.

Lemma 4.1 The number of nodes in the $(|I|+1)$-th level of $F$ is equal to $\left|\operatorname{dep}\left(f, I, x_{i}\right)\right|$.

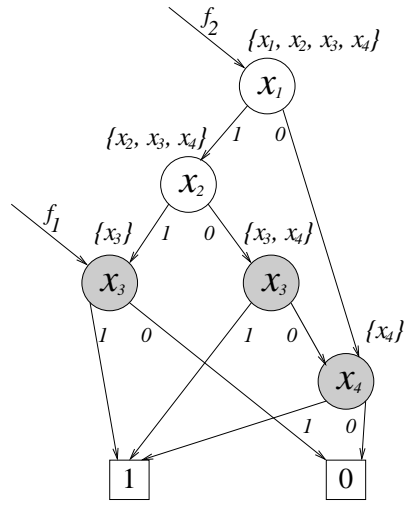

Figure 2: BDD for Example 4.1.

Example 4.1 Consider the BDD given in Figure 2. The cofactors in the variables in $I=\left\{x_{1}, x_{2}\right\}$ are represented by the grey nodes. Annotated are the sets of variables the cofactors essentially depend on. There are two cofactors depending essentially on $x_{3}$ and in fact two nodes residing on the third level for the given ordering $x_{1}<$ $x_{2}<x_{3}<x_{4}$. With the annotated sets it can be seen (without actually moving a variable), that there would also be two nodes labeled $x_{4}$ in the third level for the ordering $x_{1}<x_{2}<x_{4}<x_{3}$, since again two cofactors essentially depend on $x_{4}$.

This argument is used in step $k$ (expanding a state $I$ with $|I|=k-1$ ) of Algorithm NEO in Figure 1 as follows: Let us assume a BDD $F$ for $I$ with an ordering $\pi$ respecting $\pi(I)=I$. Computing the terms label $\left(F_{i}, x_{i}\right)$ simplifies to counting the cofactors which depend essentially on $x_{i}$. This can be done with a procedure traversing the nodes in the levels $|I|+1, \ldots, n$, which does not involve movements of variables through the BDD. Hence, no expensive graph reconstruction operations are needed anymore.

\subsection{Implementation}

Assume, the routine compute_dependencies sketched in Figure 3 is called for a BDD $F$ representing $f$ with variable ordering $\pi$ such that $\pi(I)=I$ and level $=|I|$. First, for every node $v$ in the levels level $+1, \ldots, n$ a bit mask is computed, where the bit corresponding to the index of a variable is set iff the function represented by $v$ essentially depends on this variable. The mask will represent the set of variables, the function represented by this node essentially depends on like annotated in Figure 2. This is done bottom up first considering the index of the variable, the node is labeled with (since the function represented clearly depends on that variable). Then, all bits set in the mask of the then-successor of $v$ or in the mask of the else-successor of $v$ are set in the mask of $v$ (a bitwise "or"-operation). The annotated sets in Figure 2 demonstrate the idea of this construction.

Afterwards a counter for every variable $x_{i} \in X_{n}$ is increased, iff a cofactor in all variables in $I=\left\{x_{\pi(1)}, \ldots, x_{\pi(\text { level })}\right\}$ essentially depends on $x_{i}$. This can now be tested by inspecting the bit masks of the nodes representing these cofactors (the set of these nodes is denoted cof_nodes $(F$, level $)$ in line (16) in Figure $3)$. Hence, in this array of counters, all terms $\operatorname{label}\left(F_{i}, x_{i}\right)=$ $\left|\operatorname{dep}\left(f, I, x_{i}\right)\right|$ for $x_{i} \in X_{n} \backslash I$ are computed during only one call to this routine without the computational cost of actually constructing each BDD $F_{i}$, i.e. without variable movements and the risk of the BDD size increasing. 


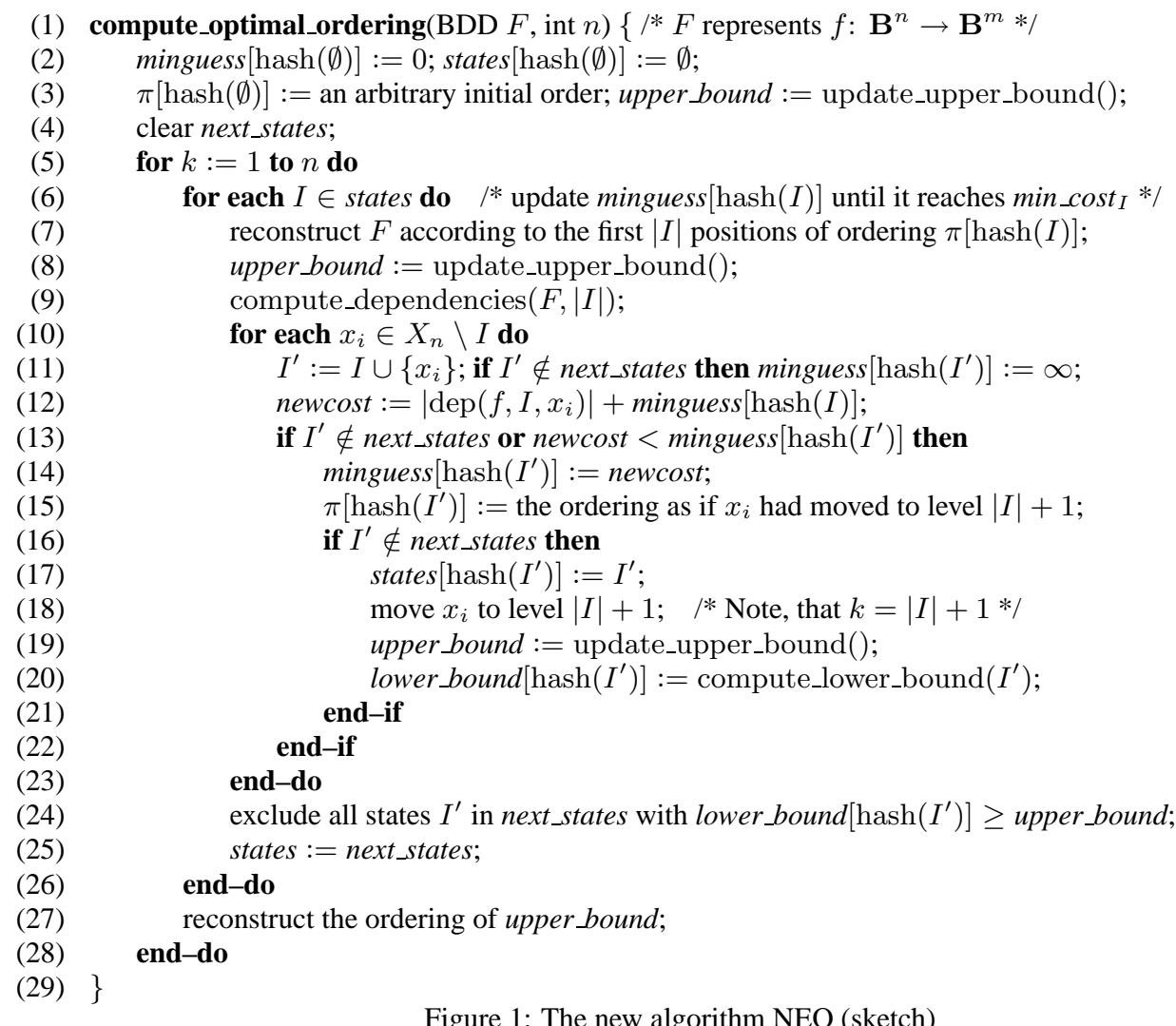

Figure 1: The new algorithm NEO (sketch)

\subsection{Revisiting States without Variable Movements}

With the new technique, all but one variable movements needed to expand all predecessor states $I$ to a particular state $I^{\prime}$ become obsolete: The only variable movement which is still left to perform only once the first time a successor state $I^{\prime}$ is encountered is the one in line (18) in Algorithm NEO in Figure 1, triggered by the conditional statement in line (16). (This movement prepares the computation of the lower bound. For more details about the computation of the lower bound see [4].) Without the new expansion technique, much more variable movements would be necessary: A successor state $I^{\prime}$ with $\left|I^{\prime}\right|=k$ is being visited up to $k$ times in the progress of one step of the minimization algorithm, since $k$ distinct states $I, J$ with $|I|=|J|=k-1$ have the successor $I^{\prime}$ in common. With the new expansion technique, revisiting $I^{\prime}$ does not involve any further variable movements.

\section{EXPERIMENTAL RESULTS}

All experimental results have been carried out on a system with an Athlon processor running at $1.4 \mathrm{GigaHz}$ using an upper memory limit of 300 MByte and a runtime limit of 20.000 CPU seconds. The new algorithm is called NEO and is compared to the best approaches to exact BDD minimization known so far, called FizZ [4] and the top-down version of JANUS [6]. All algorithms have been implemented with the CUDD package [17] and were tested in the same system environment.

In a series of experiments all algorithms were applied to the set of benchmark circuits from LGSynth93. The results are given in Table 1 . In the first column the name of the function is given.
Column $n$ denotes the number of inputs of a function. Column opt shows the number of BDD nodes needed for the minimal representation. In the columns time the runtimes in CPU seconds for FizZ, JANUS and the new approach NEO are given. The last column space shows the space requirement of the new approach NEO in MByte. Due to space limitation, this paper is focussed on the comparison of runtimes. The author remarks, that the space requirement of NEO is almost exactly the same as for JANUS given in [6].

As the results show, the new approach NEO is faster than FizZ and JANUS in almost every case. Especially for larger examples a reduction in runtime by up to $63.9 \%$ in comparison to FizZ is achieved (see e.g. mux, cm150a, cps). The gain in comparison to JANUS is up to $31.3 \%$ (see e.g. $s 820, s 832$ ). On average, the reduction in runtime is $47.5 \%$ in comparison to FizZ. The average gain compared to JANUS is $18.8 \%$. The results show that the new state expansion technique is a very robust improvement, that significantly outperforms the algorithms FizZ and JANUS.

\section{CONCLUSIONS}

A new exact algorithm for determining an optimal variable ordering for BDDs has been presented. It uses a new technique for state expansion, where time-consuming movements of variables through the BDD are not needed. Experimental results are reported that clearly demonstrate the efficiency of the presented approach. A comparison to the best minimization algorithms known so far shows that runtime can be reduced by up to $63.9 \%$. 


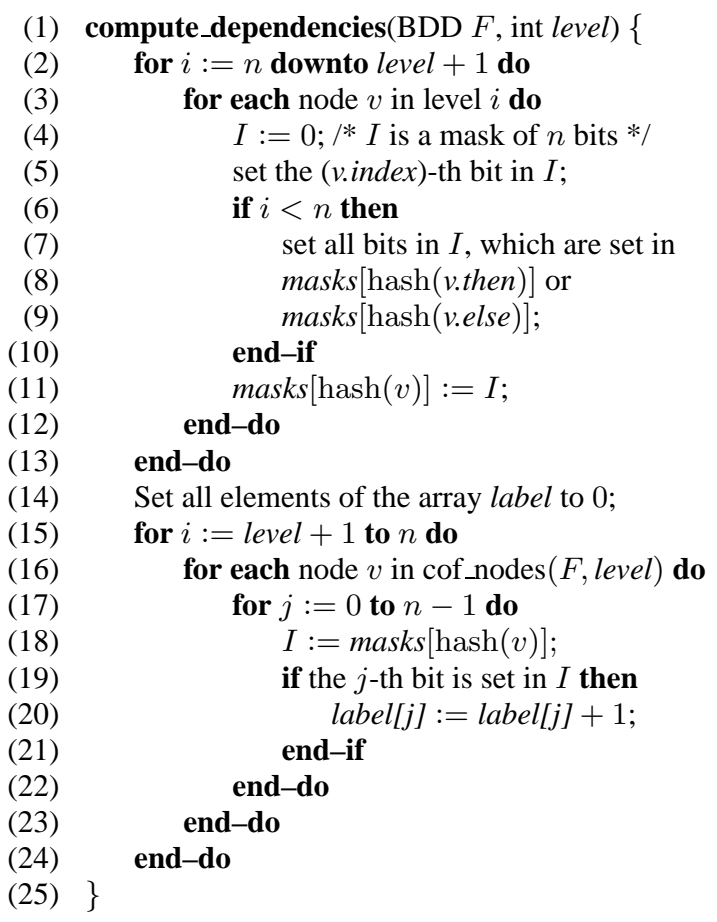

Figure 3: Computing the dependencies

\section{ACKNOWLEDGEMENTS}

The author would like to thank R. Drechsler for open-minded discussions and for a helpful introduction to BDD based approaches to logic synthesis. This work benefited much from both of that. Special thanks go to W. Günther, who supported the author with the source code of FizZ. The author also wishes to thank him for the sharing of ideas. It was W. Günther who suggested to use the BDD cache of [10] in the context of exact BDD minimization. This idea was first used in [6] and is used again in the implementation of the approach presented here. Last but not least thanks also go to P. Malik, R. Malik and O. Mayer for motivation and support.

\section{REFERENCES}

[1] B. Bollig and I. Wegener. Improving the variable ordering of OBDDs is NP-complete. IEEE Trans. on Comp., 45(9):993$1002,1996$.

[2] R. Bryant. Graph-based algorithms for Boolean function manipulation. IEEE Trans. on Comp., 35(8):677-691, 1986.

[3] P. Buch, A. Narayan, A. Newton, and A. SangiovanniVincentelli. Logic synthesis for large pass transistor circuits. In Int'l Conf. on CAD, pages 663-670, 1997.

[4] R. Drechsler, N. Drechsler, and W. Günther. Fast exact minimization of BDDs. IEEE Trans. on CAD, 19(3):384-389, 2000.

[5] R. Drechsler and W. Günther. Towards One-Pass Synthesis. Kluwer Academic Publishers, 2002.

[6] R. Ebendt, W. Günther, and R. Drechsler. Combination of lower bounds in exact BDD minimization. Accepted for DATE 2003.

[7] F. Ferrandi, A. Macii, E. Macii, M. Poncino, R. Scarsi, and F. Somenzi. Symbolic algorithms for layout-oriented syn-
Table 1: Comparison of FizZ, JANUS and NEO

\begin{tabular}{|c|c|c|c|c|c|c|}
\hline \multirow[t]{2}{*}{ name } & \multirow[t]{2}{*}{$n$} & \multirow[t]{2}{*}{ opt } & \multirow{2}{*}{$\begin{array}{l}\text { FizZ } \\
\text { time }\end{array}$} & \multirow{2}{*}{$\begin{array}{c}\text { JANUS } \\
\text { time }\end{array}$} & \multicolumn{2}{|c|}{ NEO } \\
\hline & & & & & time & space \\
\hline $\mathrm{cc}$ & 21 & 46 & $117 \mathrm{~s}$ & $84.9 \mathrm{~s}$ & $69.4 \mathrm{~s}$ & $36 \mathrm{M}$ \\
\hline $\mathrm{cm} 150 \mathrm{a}$ & 21 & 33 & $610 \mathrm{~s}$ & $311.1 \mathrm{~s}$ & $220 \mathrm{~s}$ & $37 \mathrm{M}$ \\
\hline $\mathrm{cm} 163 \mathrm{a}$ & 16 & 26 & $1.17 \mathrm{~s}$ & $0.78 \mathrm{~s}$ & $0.92 \mathrm{~s}$ & $<1 \mathrm{M}$ \\
\hline $\mathrm{cmb}$ & 16 & 28 & $0.01 \mathrm{~s}$ & $0.05 \mathrm{~s}$ & $0.33 \mathrm{~s}$ & $<1 \mathrm{M}$ \\
\hline comp & 32 & 95 & $5606 s$ & $3900 \mathrm{~s}$ & $3520 \mathrm{~s}$ & $121 \mathrm{M}$ \\
\hline cordic & 23 & 42 & $3.05 \mathrm{~s}$ & $1.82 \mathrm{~s}$ & $1.93 \mathrm{~s}$ & $<1 \mathrm{M}$ \\
\hline cps & 24 & 971 & $4396 s$ & $2751 \mathrm{~s}$ & $1893 \mathrm{~s}$ & $61 \mathrm{M}$ \\
\hline i1 & 25 & 36 & $29.4 \mathrm{~s}$ & $18.77 \mathrm{~s}$ & $22.1 \mathrm{~s}$ & $10 \mathrm{M}$ \\
\hline lal & 26 & 67 & $677 \mathrm{~s}$ & $504.4 \mathrm{~s}$ & $440 \mathrm{~s}$ & $79 \mathrm{M}$ \\
\hline $\operatorname{mux}$ & 21 & 33 & $610 \mathrm{~s}$ & $310.8 \mathrm{~s}$ & $220 \mathrm{~s}$ & $36 \mathrm{M}$ \\
\hline parity & 16 & 17 & $<0.01 \mathrm{~s}$ & $0.03 \mathrm{~s}$ & $0.27 \mathrm{~s}$ & $<1 \mathrm{M}$ \\
\hline pcle & 19 & 42 & $9.02 \mathrm{~s}$ & $5.18 \mathrm{~s}$ & $5.40 \mathrm{~s}$ & $3 \mathrm{M}$ \\
\hline pm1 & 16 & 40 & $0.55 \mathrm{~s}$ & $0.34 \mathrm{~s}$ & $0.62 \mathrm{~s}$ & $<1 \mathrm{M}$ \\
\hline s208.1 & 18 & 41 & $8.44 \mathrm{~s}$ & $5.62 \mathrm{~s}$ & $4.33 \mathrm{~s}$ & $2 \mathrm{M}$ \\
\hline s298 & 17 & 74 & $13.46 \mathrm{~s}$ & $9.06 \mathrm{~s}$ & $7.31 \mathrm{~s}$ & $2 \mathrm{M}$ \\
\hline s344 & 24 & 104 & $1446 \mathrm{~s}$ & $950 \mathrm{~s}$ & $906 \mathrm{~s}$ & $111 \mathrm{M}$ \\
\hline s349 & 24 & 104 & $1447 \mathrm{~s}$ & $950 \mathrm{~s}$ & $905 \mathrm{~s}$ & $111 \mathrm{M}$ \\
\hline s382 & 24 & 119 & $802 \mathrm{~s}$ & $461 \mathrm{~s}$ & $409 \mathrm{~s}$ & $75 \mathrm{M}$ \\
\hline s400 & 24 & 119 & $802 \mathrm{~s}$ & $456 \mathrm{~s}$ & $409 \mathrm{~s}$ & $75 \mathrm{M}$ \\
\hline s444 & 24 & 119 & $779 \mathrm{~s}$ & $508 \mathrm{~s}$ & $473 \mathrm{~s}$ & $75 \mathrm{M}$ \\
\hline s526 & 24 & 113 & $1196 \mathrm{~s}$ & $924 \mathrm{~s}$ & $705 \mathrm{~s}$ & $111 \mathrm{M}$ \\
\hline s820 & 23 & 220 & $2034 \mathrm{~s}$ & $1235 \mathrm{~s}$ & $848 \mathrm{~s}$ & $59 \mathrm{M}$ \\
\hline s832 & 23 & 220 & $2076 \mathrm{~s}$ & $1288 \mathrm{~s}$ & $889 \mathrm{~s}$ & $59 \mathrm{M}$ \\
\hline sct & 19 & 48 & $8.62 \mathrm{~s}$ & $5.97 \mathrm{~s}$ & $6.36 \mathrm{~s}$ & $3 \mathrm{M}$ \\
\hline $\mathrm{t} 481$ & 16 & 21 & $0.16 \mathrm{~s}$ & $0.13 \mathrm{~s}$ & $0.50 \mathrm{~s}$ & $<1 \mathrm{M}$ \\
\hline tcon & 17 & 25 & $0.52 \mathrm{~s}$ & $0.28 \mathrm{~s}$ & $0.79 \mathrm{~s}$ & $<1 \mathrm{M}$ \\
\hline $\mathrm{ttt} 2$ & 24 & 107 & $950 \mathrm{~s}$ & $578 \mathrm{~s}$ & $435 \mathrm{~s}$ & $82 \mathrm{M}$ \\
\hline vda & 17 & 478 & $65.4 \mathrm{~s}$ & $34.4 \mathrm{~s}$ & $32.3 \mathrm{~s}$ & $3 \mathrm{M}$ \\
\hline
\end{tabular}

thesis of pass transistor logic circuits. In Int'l Conf. on CAD, 1998.

[8] S. Friedman and K. Supowit. Finding the optimal variable ordering for binary decision diagrams. In Design Automation Conf., pages 348-356, 1987.

[9] H. Fujii, G. Ootomo, and C. Hori. Interleaving based variable ordering methods for ordered binary decision diagrams. In Int'l Conf. on CAD, pages 38-41, 1993.

[10] W. Günther and R. Drechsler. Improving EAs for Sequencing Problems. Genetic and Evolutionary Computation Conference, 175-180, 2000.

[11] N. Ishiura, H. Sawada, and S. Yajima. Minimization of binary decision diagrams based on exchange of variables. In Int'l Conf. on CAD, pages 472-475, 1991.

[12] S.-W. Jeong, T.-S. Kim, and F. Somenzi. An efficient method for optimal BDD ordering computation. In International Conference on VLSI and CAD, 1993.

[13] L. Macchiarulo, L. Benini, and E. Macii. On-the-fly layout generation for PTL macrocells. In Design, Automation and Test in Europe, pages 546-551, 2001.

[14] A. Mukherjee, R. Sudhakar, M. Marek-Sadowska, and S. Long. Wave steering in YADDs: A novel non-iterative synthesis and layout technique. In Design Automation Conf., pages 466-471, 1999.

[15] R. Murgai, R.K. Brayton, and A.L. Sangiovanni-Vincentelli. Logic Synthesis for Field-Programmable Cate Arrays. Kluwer Academic Publishers, 1995.

[16] R. Rudell. Dynamic variable ordering for ordered binary decision diagrams. In Int'l Conf. on CAD, pages 42-47, 1993.

[17] F. Somenzi. CU Decision Diagram Package Release 2.3.i. University of Colorado at Boulder, 2002. 